\title{
Hybrid strategy for unstable patients with severe carotid and cardiac disease requiring surgery
}

\author{
Oscar A. Mendiz ${ }^{1}$, Carlos M. Fava ${ }^{1}$, Gustavo A. Lev${ }^{1}$, León R. Valdivieso ${ }^{1}$, \\ Gaspar Caponi ${ }^{1}$, Gustavo F. Hidalgo Alava ${ }^{1}$, Roberto R. Favaloro ${ }^{2}$ \\ ${ }^{1}$ Department of Interventional Cardiology, University Hospital Favaloro Foundation, Buenos Aires, Argentina \\ ${ }^{2}$ Department of Cardiovascular Surgery, University Hospital Favaloro Foundation, Buenos Aires, Argentina
}

\begin{abstract}
Background: Concurrent severe carotid and cardiac disease is a challenging situation where staged surgery is probably the most common strategy. However, in patients with an unstable clinical presentation, the best approach is still a matter of debate. The aim of the study was to report in-hospital and midterm outcome in patients who received carotid artery stenting and synchronous cardiac surgery.
\end{abstract}

Methods: From June 1998 to July 2012, 54 consecutive patients who were treated at a high-volume university medical center with this hybrid approach were included in the study. All of the patients received carotid angioplasty while being administered aspirin and regular unfractionated heparin. Then, all of the patients were immediately transferred to the operating room for coronary and/or cardiac valve surgery. All of the patients were administered aspirin and clopidogrel once bleeding was ruled out, after surgery.

Results: There were 5 in-hospital surgical related deaths, and no patient suffered a stroke or required carotid urgent re-intervention. At follow-up (55 \pm 28 months; range 1-144 months), there were no new neurological deficits, while one additional death occurred.

Conclusions: In this series, synchronous carotid stenting and cardiac surgery were feasible with an acceptable complication rate in a high-surgical-risk population, which could not undergo staged procedures. (Cardiol J 2015; 22, 1: 25-30)

Key words: carotid arterial disease, carotid angioplasty, cardiac surgery

\section{Introduction}

Periprocedural stroke remains an infrequent but catastrophic complication for open heart surgery (OHS) [1]. Patients with carotid and coronary artery disease are at higher risk for stroke during coronary artery bypass grafting (CABG), with a potential benefit for those with previous carotid revascularization $[2,3]$. For stable patients, staged revascularization (carotid and then $\mathrm{CABG}$ ) seems to be the adequate strategy. However, in unstable patients, the best strategy is still under debate. Although there are few comparative studies between carotid endarterectomy
(CEA) and carotid artery stenting (CAS) previous to cardiac surgery [4], some of them showed the safety and efficacy of CAS in high risk patients [5-7].

The objective of this study is to report the clinical outcomes of patients who received simultaneous CAS and open heart surgery.

\section{Methods}

Between June 1998 and July 2013, 692 consecutive patients received CAS; $54(7.8 \%)$ of them also received a simultaneous OHS immediately after CAS, and were included in this analysis.

Address for correspondence: Dr Oscar A. Mendiz, Departamento de Cardiología Intervencionista, Hospital Universitario. Fundación Favaloro, (C1093AAS) Belgrano 1746 - 4 Piso, Ciudad Autónoma de Buenos Aires, Argentina, tel: (54 11) 4378-1359, fax: (54 11) 4378-1361, e-mail: omendiz@ffavaloro.org 
Clinical and procedural data were collected in a database for all patients and clinical and echo-Doppler follow-up was carried out for outpatients.

All patients were hospitalized due to an acute coronary syndrome and/or cardiac failure due to coronary artery disease (CAD) and/or valvular disease. All patients were unstable despite complete medical treatment and all of them were considered being at high surgical risk for the Heart Team. Their condition prevents the performing of the standard staged procedures approach, which is the most common strategy used in our hospital.

Patients with CAD cannot also be candidates for percutaneous coronary interventions. In case of valvular heart disease this should also be proven impossible to be solved by balloon valvuloplasty or other endovascular procedures when available.

CAS patients were diagnosed by echo-Doppler screening, and angiography was performed in all patients with severe lesions before therapeutic procedures.

An intervention was indicated in cases of severe stenosis; $\geq 70 \%$ in symptomatic patients and $\geq 80 \%$ in asymptomatic using the NASCET study method for measurements. All CAS stents were implanted using cerebral protection devices (CPD) after approval in January 1999.

All patients were taking aspirin $200 \mathrm{mg}$ daily; after arterial sheath insertion 10,000 IU of unfractionated heparin (UFH) was given to maintain an activated clotting time higher than $240 \mathrm{~s}$. Immediately after stenting, under UFH effect, patients were transferred from the Cath Lab to the operating room for cardiac surgery intervention. Clopidogrel was started with a loading dose when bleeding was ruled out; usually $6-8 \mathrm{~h}$ after surgery.

In patients requiring prolonged mechanical ventilation, clopidogrel was given through the nasogastric tube.

Dual antiplatelet therapy was suggested for at least 1 month and oral anticoagulation according to cardiovascular surgery.

Angiographic success was defined as $<30 \%$ residual stenosis after stent implantation and clinic success when angiographic success was achieved without a 30-day major complication (stroke, death, acute myocardial infarction [AMI], and/or urgent surgery).

All patients were evaluated by an independent neurologist before and after CAS and a new computed tomography scan was obtained in cases presenting new symptoms or neurological complication after procedures.

Minor stroke was defined when a new neurological deficit after procedures was $\leq 4$ points on the
NIH Stroke Scale at the independent neurologist evaluation; major stroke when the deficit was $\geq 4$ and transient ischemic attack (TIA) when a neurological deficit was fully recovered within $24 \mathrm{~h}$.

Neurological deficits were considered related to CAS when they occurred during CAS at any location or the ipsilateral events when they occurred after cardiac surgery, and they were related to surgery when contralateral to the stent and occurring after cardiac surgery.

Mortality was considered any death within 30 days after index procedures. AMI was defined as either a new $\mathrm{Q}$-wave or creatinine kinase-MB elevation (new Q-waves on ECG, ST elevation, or new left bundle branch block post CABG, CK-MB over $80 \mathrm{IU} / \mathrm{mL}$, new wall motion changes by echocardiography).

A 30-day combined end point was defined as: all-cause death, AMI, urgent carotid surgery, TIA or any stroke.

CAS were performed by femoral access in the majority of patients and only in a few cases by radial approach at the very end of the series. The OHS was performed according to the regular practice of the surgical team under general anesthesia and extracorporeal circulation and myocardial protection. The CAS and OHS techniques were left to the operators' discretion.

All patients signed their informed consents and accepted clinical follow-up.

\section{Statistical analysis}

Absolute numbers and percentages were used to describe the patients' population. Continuous variables are shown as mean and standard deviation. SPSS 11.0. was used for the analysis.

\section{Results}

This is a single center consecutive series of patients treated between June 1998 and July 2013 who were included in the study.

Population characteristics are the following: mean age of $70.2 \pm 8$ years, 43 patients were male, 15 diabetic, 6 had severe bilateral carotid stenosis, 29 severe lesions of the left main coronary artery, 19 severe left ventricular dysfunction (left ventricular ejection fraction $<30 \%), 10$ previous myocardial infarction, and 6 previous cardiac surgery ( 1 patient had 2 prior operations) (Table 1 ).

The standard EuroSCORE was $11.8 \pm 2.8$, Parssonet $14.4 \pm 5$, Society of Thoracic Surgeons (STS) for complications $26.2 \pm 9$, for stroke $2.7 \pm 1.3$, for STS death $3.7 \pm 0.9$ (Table 2). All patients were 
Table 1. Clinical characteristics.

\begin{tabular}{lc}
\hline Age & $70.3 \pm 8$ \\
$\geq 80$ years & $5(9.3 \%)$ \\
Male & $43(79.6 \%)$ \\
Hypertension & $50(92.6 \%)$ \\
Diabetes mellitus & $15(27.8 \%)$ \\
Smokers & $25(46.3 \%)$ \\
Dyslipidemia & $32(59.3 \%)$ \\
Previous myocardial infarction & $10(18.5 \%)$ \\
Previous CABG* & $6(11.1 \%)$ \\
Previous transient ischemic attack & $2(3.7 \%)$ \\
Previous stroke & $2(3.7 \%)$ \\
Contralateral carotid occlusion & $6(11.1 \%)$ \\
Left ventricular ejection fraction $\leq 30$ & $15(27.8 \%)$ \\
\hline
\end{tabular}

*One patient had 2 previous coronary artery bypass graftings (CABG)

Table 2. Risk score.

\begin{tabular}{lc}
\hline Parssonet & $14.4 \pm 5$ \\
EuroSCORE & $11.8 \pm 2.8$ \\
STS complications & $26.2 \pm 9$ \\
STS stroke & $2.7 \pm 1.3$ \\
STS death & $3.7 \pm 0.9$ \\
\hline
\end{tabular}

STS - Society of Thoracic Surgeons

Table 3. 30-day outcomes.

\begin{tabular}{lc}
\hline Major stroke & - \\
Minor stroke & - \\
Transient ischemic attack & - \\
Related to carotid artery stent death & - \\
Related to cardiovascular surgery death & $5(9.2 \%)$ \\
Acute myocardial infarction & $1(1.8 \%)$ \\
Bleeding requiring reoperation & $7(13 \%)$ \\
Hemodialysis & $5(9.2 \%)$ \\
\hline
\end{tabular}

unstable with a cardiac condition requiring urgent cardiac surgery and thus a previous carotid revascularization which was performed simultaneously.

\section{CAS and cardiovascular surgery}

CAS was performed by a femoral approach in 48 patients, by radial in 4 , and humeral in 2 . CPD were used in 53; 1 patient at the beginning of the series was treated without CPD. Self-expandable stents were used in all cases.

Four patients at the beginning of the series received bilateral CAS in the same setting. Angiographic success was obtained in all cases.
After CAS, 27 patients received CABG alone, 23 CABG plus aortic valve replacement (AVR), and 4 cases only valvular replacement ( 2 aortic; 1 mitral and 1 mitral plus tricuspid).

Clinical success was achieved in $49(90.7 \%)$ patients. Five patients died after surgery (4 had received combined surgery CABG plus AVR and 1 only $\mathrm{CABG}$ for the second re-operation). One death was due to surgical bleeding (a patient with 2 previous cardiovascular surgeries), 1 because a cardiogenic shock, and 3 due to multiorgan failure (Table 3).

No patient had neurological complications at 30 days.

\section{Follow-up}

The follow-up was achieved in all cases at $55 \pm 28(1-144)$ months, no patient suffered from neurological events, or any carotid or cardiovascular re-interventions. One patient died due to a non-related death (cancer).

\section{Discussion}

The association between carotid artery disease and $\mathrm{CAD}$ is a relatively frequent clinical presentation of vascular disease. About $22 \%$ of the referred patients for CABG had moderate to severe carotid artery stenosis while $30 \%$ of the referred for CEA had concomitant CAD [1].

While stroke is a devastating cardiac surgery complication and represents a challenge for cardiovascular surgeons, it is well known that carotid artery disease increases neurological complication risks for those patients undergoing CABG; these complications can be fatal and non-fatal stroke, TIA and hypoxic encephalopathy causing important physical and psychological damage to patients and their families and also increasing hospital costs. Moreover, the risk of neurological complication becomes relevant with aging patients who are referred for OHS.

In a paper from Roach et al. [8] it was reported that $6.1 \%$ of patients had a neurological deficit immediately after $\mathrm{CABG}$ and the mortality rate of these patients was $21 \%$. Thus stroke is also a condition which increases mortality rate after OHS.

Simultaneous CEA and CABG showed reducing neurological complication but increasing the mortality rate and non-fatal AMI [9]. In a study from Rome University, which included 180 consecutives patients (1991-2002) that were treated with simultaneous CEA and surgery, the mortality rate was $10.1 \%$, but in patients with EuroSCORE $\geq 6$ it was $14.5 \%$. In this setting, we thought that 
CAS currently represents one option with a lower risk for AMI in comparison to CEA.

Other report from Levy et al. [10], who used a simultaneous approach combining CEA and CABG in 80 patients, had the following perioperative outcomes: mortality rate $3.7 \%$, stroke $2.5 \%$, myocardial infarction $3.7 \%$. Combined complications of death + myocardial infarction + stroke was $10 \%$. However, CAS has shown a low myocardial infarction rate as complication leading to a lower long-term mortality rate [11].

In a recent publication from Shishehbor et al. [12], the authors analized 350 patients who received 3 different strategies: staged CEA and OHS $(\mathrm{n}=45)$, combined CAE plus OHS $(\mathrm{n}=195)$ and staged CAS plus OHS $(n=110)$. The time between CEA and OHS was as soon as possible according to clinical condition, while for CAS and OHS it was 4 weeks and 5 additional days after clopidogrel was stopped. The primary composite end point was all-cause death, stroke, and myocardial infarction. Staged CAS-OHS patients had more previous stroke and underwent more complex surgery.

Regarding the outcomes; staged CAS-OHS and combined CEA-OHS had similar early outcomes, while staged CEA-OHS had more inter-stage myocardial infarction. Subsequently, staged CAS-OHS experienced significantly fewer late hazard phase events in comparison to both staged CEA-OHS (adjusted hazard ratio [HR] 0.33; 95\% confidence interval [CI] 0.15-0.77; $\mathrm{p}=0.01$ ) and combined CEA-OHS (adjusted HR 0.35; 95\% CI 0.18-0.70; $\mathrm{p}=0.003)$. Thus the outcomes are significantly in favor of staged CAS-OHS after the first year. Thirty days and one year death and stroke rates were similar but CAS group had a lower myocardial infarction rate and better evolution [12].

CAS and CEA have shown similar outcomes in high surgical risk patients. However, there are few studies comparing these 2 revascularization strategies for patients with severe $\mathrm{CAD}$ who need urgent $\mathrm{CABG}$ or valvular surgery. Moreover, there are some reports that have shown the feasibility and safety of staged CAS and CABG [13, 14]. But, unstable patients requiring an urgent $\mathrm{OHS}$, who also have a severe carotid artery disease, represent an extremely high risk subgroup of patients, where a combined approach with a less invasive strategy, like CAS, to solve carotid artery stenosis which can increase stroke risk for OHS, with a simultaneous cardiovascular surgery, may reduce the global risk of both procedures appearing as an appealing strategy for patients with aggressive atherosclerotic disease manifestation [15].
In this present study, the hybrid, simultaneous (or synchronous) strategy (CAS and OHS) was feasible with a high but acceptable mortality rate $(9.2 \%)$ considering such a high surgical risk population without suffering from neurological complications. This is very encouraging for this highly selective group of patients who could not be staged, which would be the best strategy.

In a multicenter study; the SHARP study: 101 patients were treated with CAS and OHS and had a mortality and stroke rate of $2 \%$ [15]. This low mortality rate in comparison with our series can be explained by a different patient selection (our patients have a higher EuroSCORE $[11.8 \pm 2.8]$ than in SHARP study [8.6 \pm 2.5$])$. This is also a more recent multicenter registry than our series; thus, the learning curve and technical advances would have had an impact on the clinical outcomes.

In addition, only $14 \%$ of patients in the SHARP study underwent valvular surgery, while $50 \%$ of our cases received combined CABG plus valvular replacement and $10 \%$ only valvular surgery; 2 situations which increased the mortality risk.

New procedures and strategies like new generation drug eluting stent [16-18] and new data supporting their use in complex anatomical and clinical scenarios, and percutaneous AVR [19, 20], endovascular mitral valve repair may change the outlook for some of these patients, who can be safely treated with minimally invasive procedures.

Although the long-term results of FREEDOM [21] and SYNTAX trials [22] clearly show that multivessel disease, mainly for those with diabetes mellitus and high Syntax Score, is still better treated with CABG; in our series, 20 of our patients required combined $\mathrm{CABG}$ plus AVR representing a higher surgical risk impossible to be compared with patients included in such trials.

However, for those unstable and/or very elderly patients with valvular and/or CAD, whenever possible, the percutaneous approach appears as $\mathrm{a}$ an alternative that allows for deferring the carotid revascularization if indicated.

Although in the present study CAS outcomes were quite similar to our stable series of patients and many other similar publications, we consider that dual antiplatelet therapy represents an important part of the complexity of this challenging clinical situation and; although CAS were performed only under aspirin and UFH, second antiplatelet drug (usually clopidogrel) should be started sooner after bleeding has been ruled out in order to prevent potential carotid stent thrombosis. 
Aspirin withdrawal before OHS may decrease risk of bleeding, but this strategy still remains controversial because it would increase the risk of coronary events and inflammatory response. However, in our strategy, aspirin was kept because dual antiplatelet therapy with clopidogrel or prasugrel has been related to a higher surgical related bleeding risk and we considered it too risky to implant a carotid artery stent without any antiplatelet therapy to prevent stent thrombosis before full dual antiplatelet therapy. The role of ticagrelor which decreased complication for those patients who received CABG in PLATO study must be further investigated for this potential indication in this group of patients [23-27].

Like all other CAS patients, the cases with a hybrid approach require strict blood pressure monitoring to prevent hypotension, a main factor to drive to stent thrombosis and hypertension which may drive to the hypoperfusion syndrome.

At the beginning of our series, 4 patients received bilateral CAS without complication; however, after several publications and expert opinions about that situation describing that it may lead to a high risk of hypoperfusion syndrome [28, 29], we abandoned this strategy and in the cases with bilateral severe carotid artery stenosis we only treated the more severe one before cardiac surgery, and the other was left to the physician's decision at follow-up.

\section{Limitations of the study}

This study has several limitations, such as: a single center study without a control group, with a long period of recruitment because patients who cannot be staged are uncommon and their frequency seems to be decreasing as minimally invasive strategies are improving and becoming safer and effective. However, this is a consecutive series of patients where we used a strategy that is not so new and is still not widely accepted due to the lack of strong evidence.

\section{Conclusions}

This single center series showed that one stage hybrid strategy (CAS and simultaneous OHS) is a feasible and safe option for unstable high surgical risk patients with concomitant severe carotid and coronary and/or cardiac valvular disease requiring cardiovascular surgery that cannot be staged.

Conflict of interest: None declared

\section{References}

1. Naylor AR, Mehta Z, Rothwell PM, Bell PR. Carotid artery disease and stroke during coronary artery bypass: A critical review of the literature. Eur J Vasc Endovasc Surgery, 2002; 23: 283-294.

2. Fareed KR, Rothwell PM, Mehta Z, Naylor AR. Synchronous carotid endarterectomy and off-pump coronary bypass: An updated, systematic review of early outcomes. Eur J Vasc Endovasc Surgery, 2009; 37: 375-378.

3. Hirotani T, Kameda T, Kumamoto T, Shirota S, Yamano M. Stroke after coronary artery bypass grafting in patients with cerebrovascular disease. Ann Thoracic Surgery, 2000; 70: 1571-1576.

4. Ziada KM, Yadav JS, Mukherjee D et al. Comparison of results of carotid stenting followed by open heart surgery versus combined carotid endarterectomy and open heart surgery (coronary bypass with or without another procedure). Am J Cardiol, 2005; 96: 519-523.

5. Fairman R, Gray WA, Scicli AP et al. The capture registry: Analysis of strokes resulting from carotid artery stenting in the post approval setting: Timing, location, severity, and type. Ann Surg, 2007; 246: 551-556; discussion 556-558.

6. Gray WA, Hopkins LN, Yadav S et al. Protected carotid stenting in high-surgical-risk patients: The archer results. J Vascular Surgery, 2006; 44: 258-268.

7. Yadav JS, Wholey MH, Kuntz RE et al. Protected carotid-artery stenting versus endarterectomy in high-risk patients. New Engl J Med, 2004; 351: 1493-1501.

8. Roach GW, Kanchuger M, Mangano CM et al. Adverse cerebral outcomes after coronary bypass surgery. Multicenter study of perioperative ischemia research group and the ischemia research and education foundation investigators. New Engl J Med, 1996; 335: 1857-1863.

9. Naylor AR, Mehta Z, Rothwell PM, Bell PR. Reprinted article "carotid artery disease and stroke during coronary artery bypass: A critical review of the literature". Eur J Vasc Endovas Surgery, 2011; 42 (suppl. 1): S73-S83.

10. Levy E, Yakubovitch D, Rudis E et al. The role of combined carotid endarterectomy and coronary artery bypass grafting in the era of carotid stenting in view of long-term results. Interact Cardiovasc Thorac Surg, 2012; 15: 984-988.

11. Roffi M, Cremonesi A. Current concepts on the management of concomitant carotid and coronary disease. J Cardiovasc Surg (Torino), 2013; 54: 47-54.

12. Shishehbor MH, Venkatachalam S, Sun Zet al. A direct comparison of early and late outcomes with three approaches to carotid revascularization and open heart surgery. J Am Coll Cardiol, 2013; 62: 1948-1956.

13. Van der Heyden J, Suttorp MJ, Bal ET et al. Staged carotid angioplasty and stenting followed by cardiac surgery in patients with severe asymptomatic carotid artery stenosis: Early and long-term results. Circulation, 2007; 116: 2036-2042.

14. Guzman LA, Costa MA, Angiolillo DJ et al.A systematic review of outcomes in patients with staged carotid artery stenting and coronary artery bypass graft surgery. Stroke, 2008; 39: 361-365. 
15. Versaci F, Reimers B, Del Giudice C et al. Simultaneous hybrid revascularization by carotid stenting and coronary artery bypass grafting: The sharp study. J Am Coll Cardiol, 2009; 2: 393-401.

16. Brugaletta S, Garcia-Garcia HM, Onuma Y, Serruys PW. Everolimuseluting absorb bioresorbable vascular scaffold: Present and future perspectives. Expert Review Medical Devices, 2012; 9: 327-338.

17. Garg S, Serruys P, Onuma Y et al. 3-year clinical follow-up of the xience $\mathrm{v}$ everolimus-eluting coronary stent system in the treatment of patients with de novo coronary artery lesions: The spirit ii trial (clinical evaluation of the xience v everolimus eluting coronary stent system in the treatment of patients with de novo native coronary artery lesions). J Am Coll Cardiol Cardiovascular Interventions, 2009; 2: 1190-1198.

18. Silber S, Windecker S, Vranckx P, Serruys PW. Unrestricted randomised use of two new generation drug-eluting coronary stents: 2 -year patient-related versus stent-related outcomes from the resolute all comers trial. Lancet, 2011; 377: 1241-1247.

19. Kodali SK, Williams MR, Smith CR et al. Two-year outcomes after transcatheter or surgical aortic-valve replacement. New Engl J Med, 2012; 366: 1686-1695.

20. Makkar RR, Fontana GP, Jilaihawi $\mathrm{H}$ et al. Transcatheter aortic valve replacement for inoperable severe aortic stenosis. New Engl J Med, 2012; 366: 1696-1704.

21. Farkouh ME, Domanski M, Sleeper LA et al. Strategies for multivessel revascularization in patients with diabetes. N Engl J Med, 2012; 367: 2375-2384.

22. Serruys PW, Morice MC, Kappetein AP et al. SYNTAX Investigators. Percutaneous coronary intervention versus coronary artery bypass grafting for severe coronary artery disease. $\mathrm{N} \mathrm{Engl}$ J Med, 2009; 360: 961-972. Erratum in: N Engl J Med, 2013; 368: 584 .
23. Berg K, Langaas M, Ericsson M et al. Acetylsalicylic acid treatment until surgery reduces oxidative stress and inflammation in patients undergoing coronary artery bypass grafting. Eur J Cardiothorac Surg, 2013; 43: 1154-1163.

24. Miceli A, Duggan SM, Aresu G et al. Combined clopidogrel and aspirin treatment up to surgery increases the risk of postoperative myocardial infarction, blood loss and reoperation for bleeding in patients undergoing coronary artery bypass grafting. Eur J Cardiothorac Surg, 2013; 43: 722-728.

25. Cannon CP, Harrington RA, James S et al.; PLATelet inhibition and patient Outcomes. Comparison of ticagrelor with clopidogrel in patients with a planned invasive strategy for acute coronary syndromes (PLATO): A randomised double-blind study investigators. Lancet, 2010; 375: 283-293.

26. Montalescot G, Wiviott SD, Braunwald E et al. Prasugrel compared with clopidogrel in patients undergoing percutaneous coronary intervention for ST-elevation myocardial infarction (TRITON-TIMI 38): Double-blind, randomised controlled trial. TRITON-TIMI 38 Investigators. Lancet, 2009; 373: 723-731.

27. Becker RC, Bassand JP, Budaj A et al. Bleeding complications with the P2Y12 receptor antagonists clopidogrel and ticagrelor in the PLATelet inhibition and patient Outcomes (PLATO) trial. Eur Heart J, 2011; 32: 2933-2944.

28. Henry M, Gopalakrishnan L, Rajagopal S, Rath PC, Henry I, Hugel M. Bilateral carotid angioplasty and stenting. Catheterization Cardiovasc Interventions, 2005; 64: 275-282.

29. Meyers PM, Higashida RT, Phatouros CC et al. Cerebral hyperperfusion syndrome after percutaneous transluminal stenting of the craniocervical arteries. Neurosurgery, 2000; 47: 335-343; discussion 343-335. 